\title{
STUDY ON THE EFFECTS OF PREHEATED WALL/PLATES IN MICROTHRUSTER SYSTEMS
}

\author{
Elyas Lekzian, Hamid Parhizkar, Asghar Ebrahimi \\ Aerospace Faculty, Malek Ashtar University of Technology, Tehran, Iran \\ e-mail:lekzian@mut.ac.ir; hparhiz@mut.ac.ir; ebrahimi@mut.ac.ir
}

\begin{abstract}
In the present paper, effects of pre-heated walls/plates on microthrusters performance are studied using a DSMC/NS solver. Three microthruster configuration types are studied. Type 1 is a cold gas microthrster. Microthruster type 2 has pre-heated walls. Pre-heated plates are inserted inside the chamber of microthruster type 3. It is observed that in microthruster type 2 the flow is accelerated and the specific impulse is elevated. However, by insertion of the pre-heated plates in microthruster type 3, viscous effects have stronger negative influence and the thrust is decreased. By implementing temperature gradients on walls in type 2 and on plates in type 3 , it is observed that a higher temperature gradient enhances performance parameters of microthruters. Among all types of microthrusters, microthruster type 2 with pre-heated walls has the highest thrust and specific impulse. Microthruster type 3 with a temperature gradient of $300-500 \mathrm{~K}$ has the minimum thrust due to a considerable decrease in the mass flow rate.
\end{abstract}

Keywords: DSMC/NS solver, pre-heated walls/plates, microthruster, performance parameters, temperature gradient

\section{Introduction}

Lots of micro devices such as microchannels, micro heat sinks, microturbines, microengines, and microthrusters were developed with advancement in facbrication. Due to advantages of micro-electro-mechanical-systems (MEMS) compared to their macro counterparts, they find increased applications in a variety of industrial and medical fields (Gad-el-Hak, 2005a,b). These miniaturized devices utilize smaller volumes while offering the possibility of parallel operation on a chip and reduce the risk of the whole system failure. In addition to advantages of scale miniaturization, they utilize little energy, offer high sensitivity and work with great accuracy. Besides the present applications of MEMS in electrical, structural, fluidic, transport and control aspects (Gad-el-Hak, 2005a,b), the potential applications such as attitude control of small satellites using micro thrusters in deep space (Janson et al., 1999; Platt, 2002; Osiander et al., 2005; Mihailovic et al., 2011) are a field of interest.

In 1960s, low thrust devices were analyzed experimentally (Milligan, 1964; Rothe, 1971). Then numerical methods were proposed by Rae (1971) and Boyd et al. (1992) in order to investigate converging-diverging nozzles numerically. Boyd used a DSMC method and proved that this method was accurate enough to analyze the flow especially in the expanding section of the thruster with a near vacuum exit boundary conditions. Small nozzles were studied by Bayt (1999). He modeled a MEMS based nozzle by using Navier-Stokes simulations and observed that by a decrease of Reynolds number the propulsive efficiency was decreased due to an increase in boundary layer thickness. A microthruster utilized in GP-B spacecraft was studied by Jafryt and Beukelt (1994). They studied back pressure effects on the behavior of flow in the nozzle. Ivanov et al. (1999) studied the effects of the throat Reynolds number on the specific impulse. They demonstrated that an increase of losses could led to over-prediction of the specific impulse at 
the exit of the nozzle. Alexeenko et al. (2002) investigated an axisymmetric 3D micronozzle by the DSMC method and Navier-Stokes solver. Their results showed that gas expansion increased the specific impulse. Xie (2007) demonstrated that flow in MEMS nozzles was simulated accurately by the DSMC method and the Navier-Stokes equations even when adding slip boundary conditions became invalid when the average Knudsen number was about 0.01 in his special case. Surface roughness of the micro nozzle was studied by Torre et al. (2010). They observed shocks near the walls due to roughness. Sun et al. (2009) conducted a DSMC-FVM method to simulate the flow inside a micro nozzle. They investigated the effect of inlet pressure on the flow field inside the nozzle. They concluded that when the inlet pressure was increased, the distance between the throat and the point that propellant velocity surpassed sonic velocity became smaller.

In the present study, pressure driven microthrusters are investigated. Numerical simulation of the flow passing through the thruster is introduced first. A DSMC/NS solver is utilized. Due to lack of experimental data at microscales, the solver is first verified by a simulated micronozzle. The solver is then utilized to simulate microthrusters. Effects of heating the flow by increasing the wall temperature and by insertion of high temperature plates inside the domain are studied, and performance parameters are then investigated. Next, the effects of the temperature gradient on the performance parameters are analyzed. At the end, the thrust and specific impulse of all microthrusters configurations are compared.

\section{Basic theory}

\subsection{DSMC solver}

DSMC method enables numerical solution of the Boltzmann equations. The method benefits from the statistical solution of the particles behavior represented by kinetic theory (Bird, 1994).

For an accurate DSMC simulation, four issues must be considered: The cell size, time step, number of particles per cell and the mean collision separation distance. The cell size must be $1 / 3$ of the mean free path (Hadjiconstantinou, 2000; Pfeiffer et al., 2013) to prevent errors in diffusion. In the present study, the method proposed by Nance et al. (1998) is utilized to determine the grid distribution. The time step must be smaller than the mean collision time in order to properly distinguish the particle free movement from a pair collision. In order to obtain physical and meaningful solutions, Bird (2007) propose that the particle per cell (PPC) would be around 7. However, Le et al. (2006) showed that for the low speed portion of the flow near the inlet of the computational domain, an average of 20 particles per cell gives precise solutions. In this paper, the study is begun with $\mathrm{PPC}=7$, and then a PPC independence study is conducted. Another parameter that affects the results is the mean collision separation distance. According to Moss and Bird (2005), the mean separation distance has to be smaller than the mean free path in each cell throughout the computational domain.

The DSMFOAM solver of OPENFOAM-2.3.1 is used in DSMC simulations. The boundary condition proposed by Liu et al. (2006) is applied in simulation. The boundary condition in microchannel systems strongly affects the results, as described by Nance et al. (1998). Current boundary treatment is simple and stable. Many publications (Liou and Fang, 2000; Wang and Li, 2004; Roohi et al., 2009) demonstrated the accuracy of this boundary condition. The solver is capable of parallel operation and can model any geometry and any number of gas species. For calculation of post collision velocities and modeling of particle collisions, the Variable Hard Sphere (VHS) model is used. This model is widely applied because of its simplicity and good approximation of intermolecular collisions (Le et al., 2006). 


\subsection{NS solver}

By using the Chapman-Enskog procedure, the Navier stokes equations can be derived from the Boltzmann equations. The conservation of mass, momentum and energy are used (OPENFOAM, 2014). Maxwell velocity slip boundary conditions including thermal creep effects are implemented on the walls and plates (O'Hare et al., 2007)

$$
\mathbf{V}_{g}-\mathbf{V}_{w}=-\frac{2-\sigma_{u}}{\sigma_{u}} \frac{\lambda}{\mu} \boldsymbol{\tau}-\frac{3}{4} \frac{\operatorname{Pr}(\gamma-1)}{\gamma p} \mathbf{q}
$$

where $\sigma_{u}$ is the momentum accommodation coefficient. Subscripts $g$ and $w$ denote the gas adjacent to the wall and the wall, respectively. The parameter $\gamma$ is the specific heat ratio and Pr is the Prandtl number. The shear stress is $\boldsymbol{\tau}=\mathbf{S} \cdot(\mathbf{n} \cdot \mathbf{\Pi})$ and the heat flux is $\mathbf{q}=\mathbf{Q} \cdot \mathbf{S}$. The tensor $\mathbf{S}=\mathbf{I}-\mathbf{n n}$, where $\mathbf{I}$ is the identity tensor, and $\mathbf{n}$ is the normal unit vector to the wall. $\mathbf{Q}$ is the heat flux vector along the wall, and $\mathbf{\Pi}$ is the stress tensor at the wall. The parameter $\mu$ is the gas viscosity and can be calculated using both the power law and the Sutherland law. The power law model is slightly less accurate than the Sutherland law at moderate temperatures. Therefore, the Sutherland law is used for calculation of gas viscosity as follows (Le and Roohi, 2015)

$$
\mu=A_{S} \frac{\sqrt{T^{3}}}{T+T_{S}}
$$

The parameter $A_{S}$ and $T_{S}$ are constants, $A_{S}=1.41 \cdot 10^{-6} \mathrm{Pas} / \mathrm{K}^{2}, T_{S}=111 \mathrm{~K}$ for nitrogen. Maxwellian definition can be used for calculation of the molecular mean free path $\lambda$ (Le et al., 2012), but for the VHS collision model the molecular mean free path can be calculated as follows (Bird, 1994)

$$
\lambda=\frac{2(5-2 \omega)(7-2 \omega)}{15} \sqrt{\frac{m}{2 \pi k T} \frac{\mu}{\rho}}
$$

where $\omega$ is the temperature exponent of the viscosity coefficient in the power law viscosity model, $\omega=0.74$ for nitrogen (Bird, 1994). The parameter $m$ is molecular mass, and $k$ is Boltzmann constant. It has been observed that the temperature of the rarefied gas adjacent to the wall is not equal to the wall temperature. Therefore, temperature jump boundary conditions are implemented on the walls when a dilute gas is simulated by the NS equations. In the present study, the second order Smoluchowski temperature jump boundary condition is used as follows (Karniadakis et al., 2006)

$$
T_{g}-T_{w}=-\frac{2-\sigma_{T}}{\sigma_{T}} \frac{2 \gamma}{\gamma+1} \frac{1}{\operatorname{Pr}} \lambda \frac{\partial T}{\partial n}-\frac{2-\sigma_{T}}{\sigma_{T}} \frac{2 \gamma}{\gamma+1} \frac{1}{\operatorname{Pr}} \frac{\lambda^{2}}{2} \frac{\partial^{2} T}{\partial n^{2}}
$$

where $\sigma_{T}$ is the thermal accommodation coefficient and $n$ is the unit vector normal to the wall. Momentum and thermal accommodation coefficients are equal to unity in order to simulate diffuse reflector walls.

For NS simulation of the flow, RhoCentralFoam solver in OPENFOAM-2.3.1 is used. RhoCentralFoam is a density-based compressible flow solver based on central-upwind schemes of Kurganov and Tadmor.

\section{Solver algorithm}

To make use of the DSMC/NS solver, a parameter is required to be defined in order to distinguish the continuum and molecular regions. In the present study, the local Knudsen number is utilized to distinguish rarefied and continuum regions. The local Knudsen number is defined as below 


$$
\mathrm{Kn}_{\text {local }}=\frac{\lambda_{\text {local }}}{\varphi\left(\frac{\Delta \varphi}{\Delta x}\right)^{-1}}
$$

where $\varphi$ is one of the macroscopic properties in each cell including: velocity, temperature, or density. In the NS solver, the parameter $\lambda_{\text {local }}$ is calculated using equation (2.3). In the DSMC solver, the local mean free path is determined as below (Bird, 1994)

$$
\lambda_{\text {local }}=\frac{1}{\sqrt{2} \pi d^{2} n}
$$

where $d=4.17 \cdot 10^{-10} \mathrm{~m}$ is the nitrogen molecular diameter, and $n$ is the density number within each cell. The local Knudsen number is determined as follows: $\mathrm{Kn}_{\text {local }}=$ $\max \left(\mathrm{Kn}_{\text {local }}, \mathrm{Kn}_{\text {local }_{u}}, \mathrm{Kn}_{\text {local }}\right.$ ) (Boyd et al., 1995). Based on the procedure proposed by Schwartzentruber et al. (2007), the DSMC/NS solver simulation cycle is as follows:

- Entire computational domain is solved by the NS solver,

- Local Knudsen number is calculated and the continuum and rarefied regions are distinguished,

- Rarefied regions are solved by the DSMC solver,

- Local Knudsen number is recalculated, and the interface of the continuum and rarefied region is changed,

- Steps 3 and 4 are repeated until location of the interface region does not change.

\section{Validation}

\subsection{DSMC solver validation}

There is no experimental study on microthruster systems at micro scales. Therefore, in order to verify the accuracy of the DSMC solver, simulation results are compared with Liu et al. (2006). After a careful grid study based on the method proposed by Nance et al. (1998), the domain is divided into 400 cells in the $x$ direction and 150 cells in the $y$ direction by using a structured grid. The temperature and Mach number contours are depicted in Fig. 1a. The centerline temperature and Mach number are presented in Fig. 1b, and the results are compared with those presented in Liu et al. (2006).

Considering that the mean separation distance between the simulation particles must be smaller than the mean free path in each cell, two different particles per cell (PPC) $(\mathrm{PPC}=15$, PPC $=20)$ are chosen. As seen in Fig. 1b, a similar Mach number and temperature distribution is achieved. Therefore, correct simulation results are obtained by both $\mathrm{PPC}=15$ and $\mathrm{PPC}=20$. Also, agreements of the results with those of Liu et al. (2006) shows that the DSMC solver is accurate and the results are trustworthy.

\subsection{DSMC/NS solver validation}

In the previous Section, the entire domain is solved by the DSMC solver. In this Section, the DSMC/NS solver is used to obtain the solution. As mentioned earlier, the local Knudsen number needs to be determined. The domain is divided into $400 \times 150$ cells using a structured grid (Fig. 2a). Figure 2b shows the density based local Knudsen number which is the maximum local Knudsen number. It is observed that the boundary layer region in the divergent section of the micronozzle is in the rarefied regime since the local Knudsen number in this part is more than 0.05 (Boyd et al., 1995). Due to expansion of flow in the divergent section, the rarefied region is increased toward the end of nozzle. It is demonstrated by Darbandi and Roohi (2011) that solving the flow using the NS solver in the divergent section is not accurate even in the 
(a)

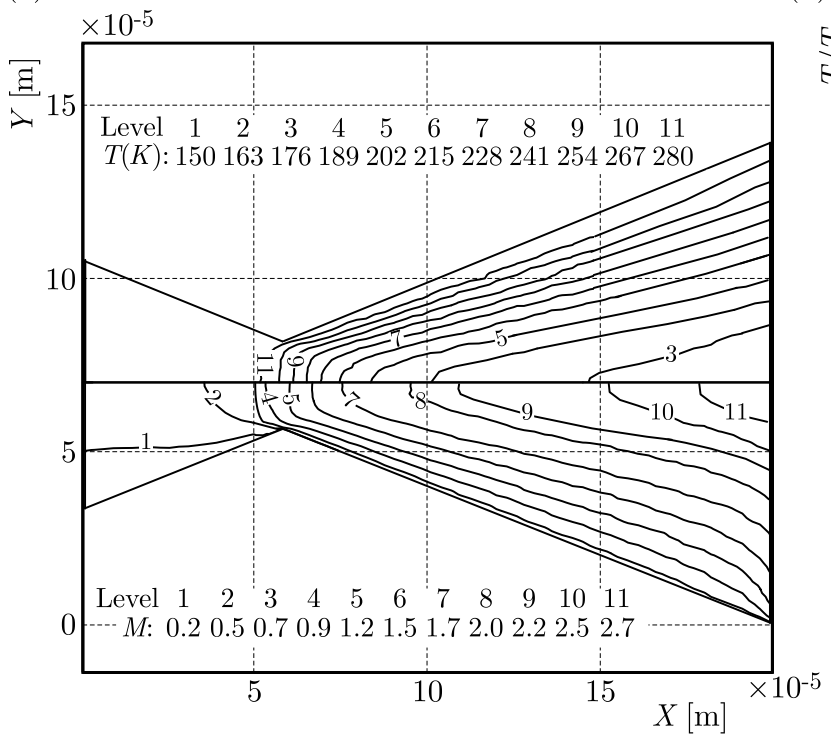

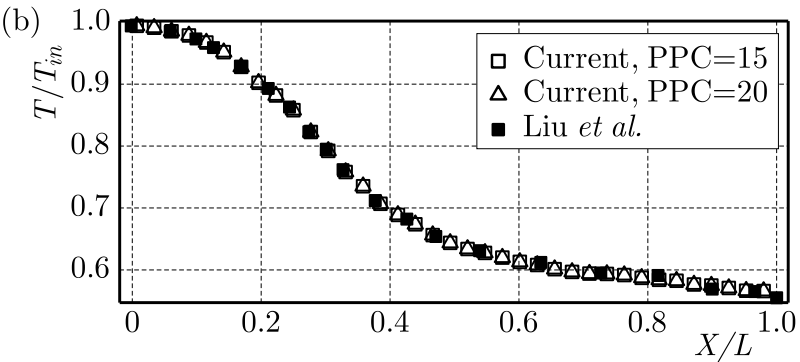

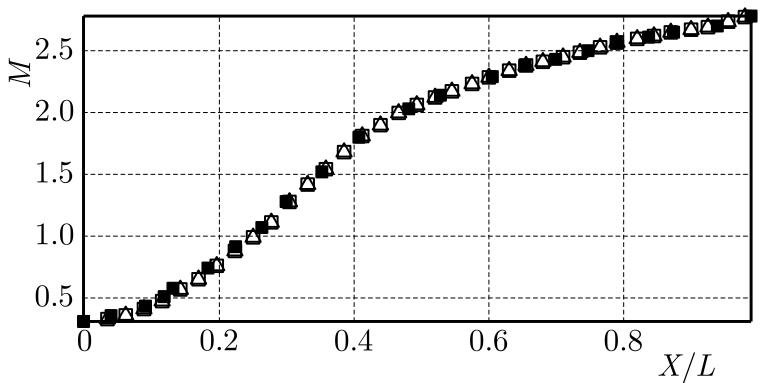

Fig. 1. (a) Temperature, Mach contours and graphs of the micronozzle; (b) current temperature and Mach number compared with Liu et al. (2006)

centerline. Therefore, the DSMC solver is used to simulate the entire divergent section and the NS solver is used for simulation of the convergent section of the micronozzle. The convergent section of the domain is divided into $100 \times 150$ cells and the divergent section is divided into $300 \times 150$ cells. Careful grid study is carried out based on the reference (Nance et al., 1998) to choose pre-mentioned grid division of the domain. Pressure and temperature are considered as inlet boundary conditions of the micronozzle at the convergent section. The solution at the end of the convergent section is considered as the inlet boundary conditions of the divergent section. This data is considered as the initial data for calculation of pressure, temperature and flux by averaging over the inlet of the divergent section.

(a)

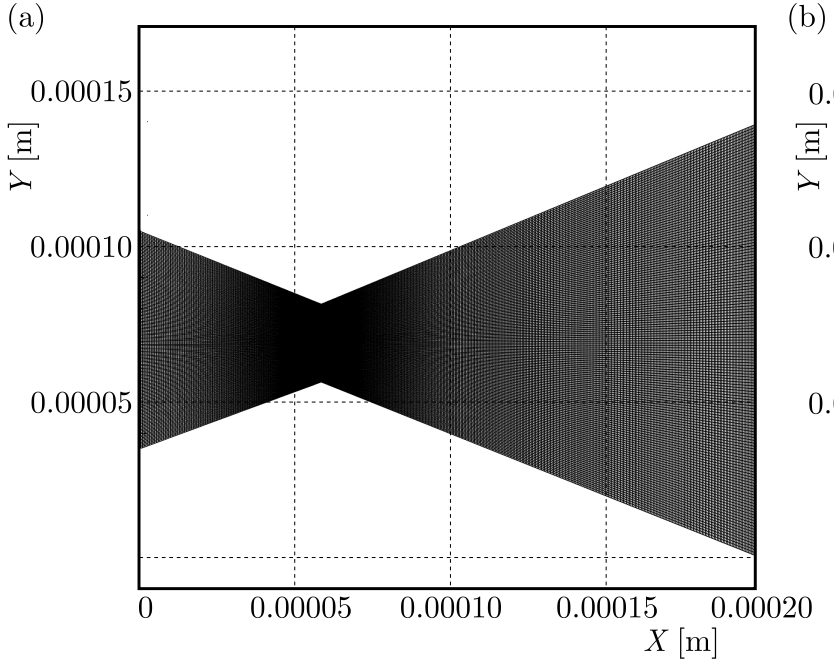

(b)

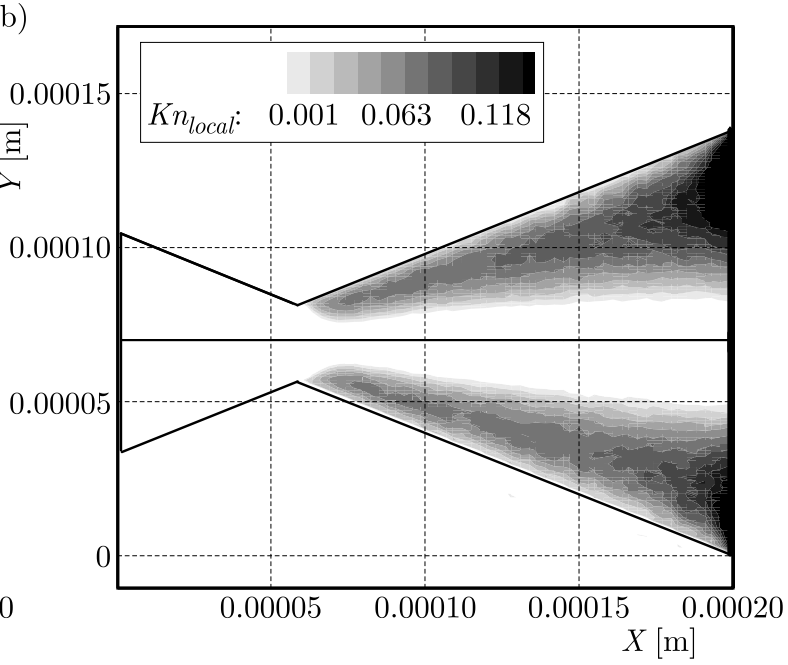

Fig. 2. Grid and Knudsen number distribution of the micronozzle: (a) structured grid, (b) Knudsen number contours using the DSMC solver (up) and the NS solver (bottom)

Figure 3 shows temperature distribution along the micronozzle centerline achieved by the DSMC and DSMC/NS solvers. 


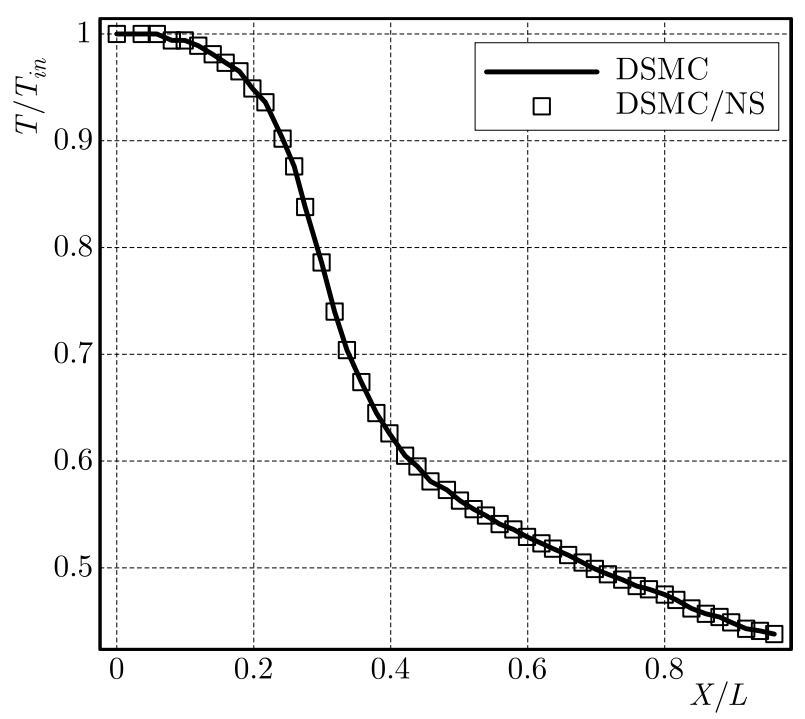

Fig. 3. Temperature distribution using the DSMC and DSMC/NS

It is observed that the result of the DSMC/NS solver is exactly the same as of the DSMC solver. Although the domain can be solved by the DSMC solver, the DSMC/NS solver is developed to reduce computational cost. DSMC simulation of the above micronozzle (using an Intel Core i5 computer with 3 GB RAM) takes 35 hours while using the DSMC/NS solver the time of computations is reduced to 23 hours (21 hours for the DSMC solver and 2 hours for the NS solver).

\section{Microthruster problem statement}

In this Section, the DSMC/NS solver is used to simulate microthruster systems. The chosen microthruster device is shown in Fig. 4. The thruster consists of a rectangular channel which is connected to a converging-diverging nozzle. A buffer zone is attached to the end of the diverging nozzle to consider variation of parameters at the nozzle exit (Wu et al., 2001).

Three types of microthrusters are considered. In type 1, a cold gas microthruster is simulated (Fig. 4 without heater plates). Type 2 is the case when the flow is heated by increasing the wall temperature up to $900 \mathrm{~K}$ (see Fig. 4 without heater plates, the wall temperature is $900 \mathrm{~K}$ ). The increasing of the wall temperature can be performed by using hot wire coated walls (Kundu et al., 2013). For type 3, heater plates (with temperature $900 \mathrm{~K}$ ) are inserted into the thruster (see Fig. 4 with heater plates). The overall geometry (but not the exact dimensions), the structure and idea of heating the fluid flow in type 3 is adapted from Kundu et al. (2013), Hitt et al. (2001) but not exactly with the same components. They fabricated and tested such a microthruster. Geometrical details of the three types of microthrusters are mentioned in Table 1.

Table 1. Dimensional parameters of the three types of microthrusters

\begin{tabular}{|c|c|c|c|c|c|c|c|c|}
\hline & $L_{1}$ & $L_{2} / L_{1}$ & $L_{3} / L_{1}$ & $L_{4} / L_{1}$ & $L_{5} / L_{1}$ & $H_{1}$ & $H_{2} / H_{3}$ & $H_{3} / H_{1}$ \\
\hline$\overline{\text { Type } 1}$ & \multirow{3}{*}{$0.6 \mathrm{~mm}$} & \multirow{3}{*}{0.4} & \multirow{3}{*}{0.62} & \multirow{3}{*}{0.83} & $=$ & \multirow{3}{*}{$0.3 \mathrm{~mm}$} & \multirow{3}{*}{0.5} & \multirow{3}{*}{0.33} \\
\hline Type 2 & & & & & 0.17 & & & \\
\hline Type 3 & & & & & 0.17 & & & \\
\hline
\end{tabular}

The gas inside the thrusters is nitrogen. In all types of the thrusters mentioned above, the inlet temperature of $300 \mathrm{~K}$ is imposed. Since the velocity at the thruster exit is supersonic, no outlet pressure is set at the exit. 


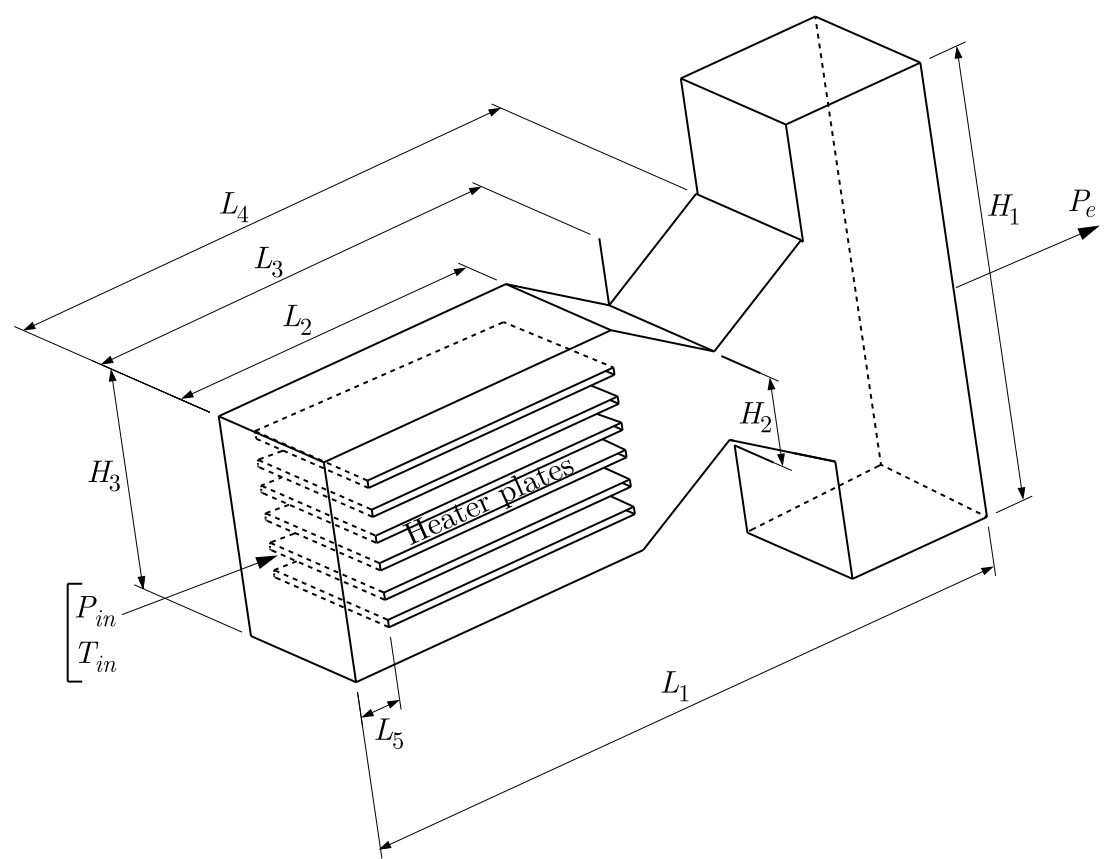

Fig. 4. Geometry of the microthruster

\subsection{Flow properties comparison of type 1, 2, and 3}

Temperature contours for the three types of microthrusters are shown in Fig. 5. Type 1 is considered as the reference case. In type 1, the temperature is decreased in the divergent section due to gas expansion. In type 2, the temperature is increased and reaches to the maximum value (less than $900 \mathrm{~K}$ ) in the centerline and then it decreases. In type 3 , the temperature is increased (to the maximum temperature of $900 \mathrm{~K}$ of pre-heated plates), it remains constant in the pre-heated plates section and then is decreased.

(a)

\section{$T[\mathrm{~K}]$ :}

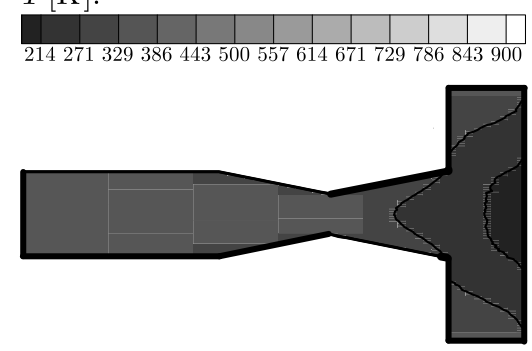

(b)

\section{$T[\mathrm{~K}]$ :}

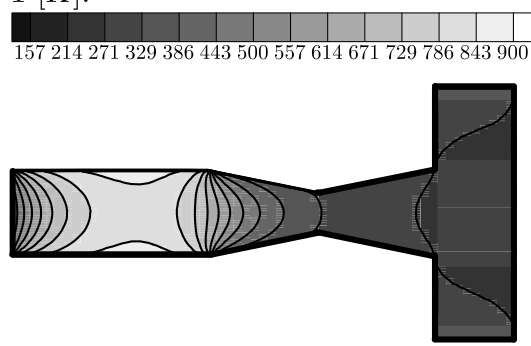

(c)

\section{$T[\mathrm{~K}]$ :}

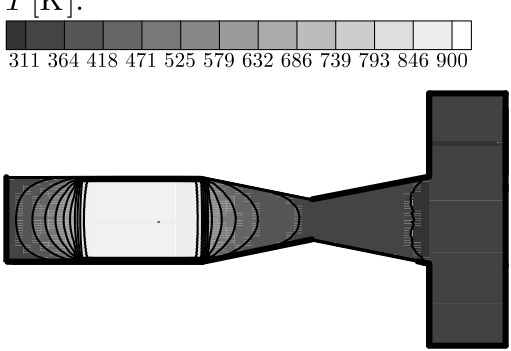

Fig. 5. Temperature contours for three types of microthrusters: (a) type 1 - cold gas, (b) type $2-$ pre-heated walls, (c) type 3 - pre-heated plates

For accurate analysis of types 1,2 , and 3 , the flow properties along the microthruster centerline are presented and compared in Fig. 6. These graphs illustrate the effect of pre-heated walls/plates inside the device on the gas flow properties. A significant pressure reduction occurs when the flow passes over heater plates in type 3 while the pressure along the centerline changes more smoothly in type 2 and 1 . At the beginning and at the end of the heater plates the temperature is increased and decreased rapidly in type 3 , whereas the temperature is increased more smoothly in type 2 . In the convergent section, it is seen that the heater plates of type 3 microthruster system affect the downstream temperature flow field and increase the temperature downstream the plates. It can be concluded that the pressure loss at the heater plates in type 3 is converted into a temperature increase. 

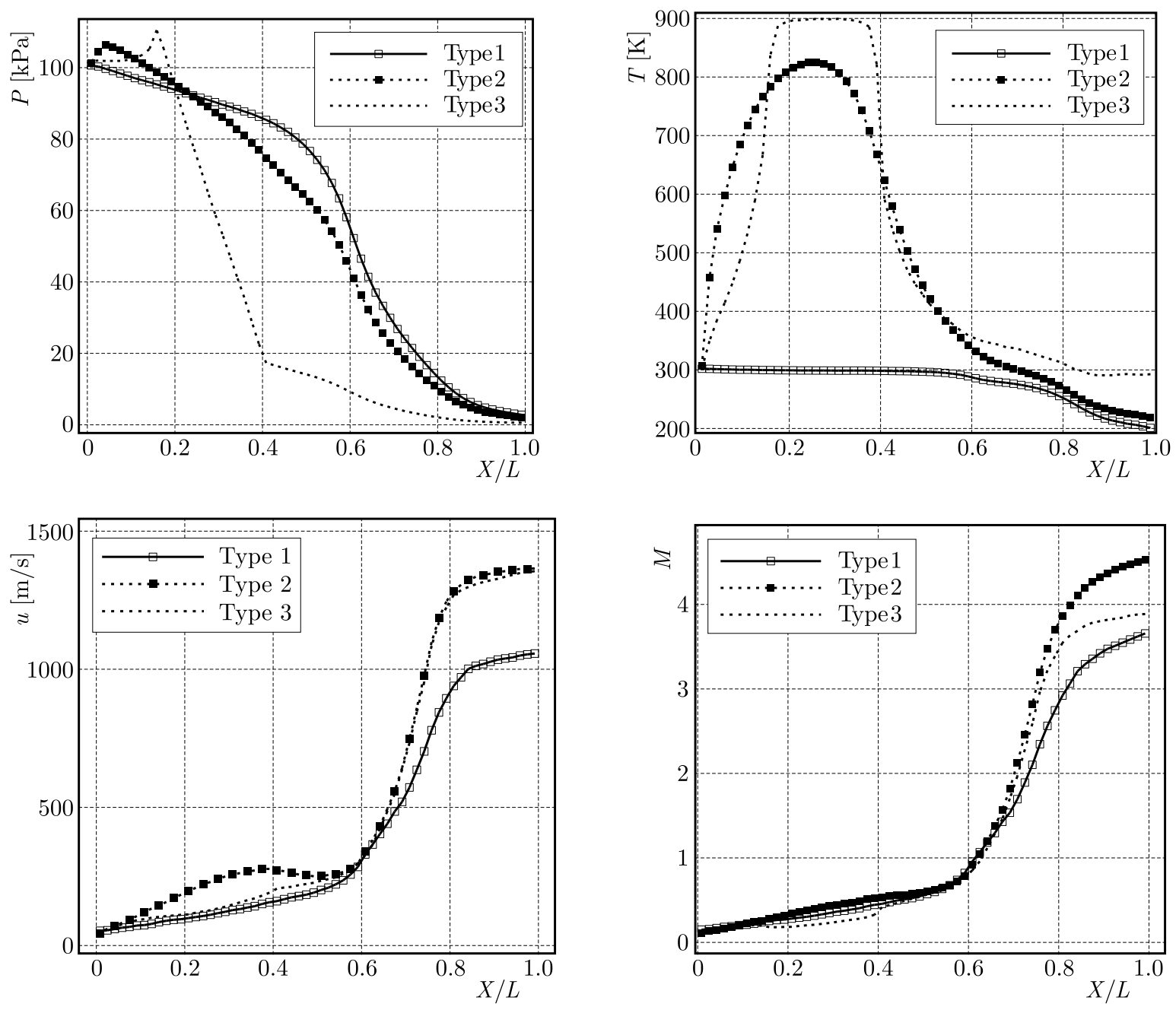

Fig. 6. Flow properties comparison of three types of microthrusters

The temperature at the diverging section of the micro propulsion device type 2 is almost similar to the cold gas microthruster. The exit velocity for type 2 and 3 is identical and greater than type 1 . Therefore, the heating process accelerates the flow. Heating the flow also affects the Mach number at the exit. In type 2 and 3, the choking place of flow is not changed, but the exit Mach number of type 2 is greater than type 3. Such behavior is expected because the flow velocity at the exit is almost identical for type 2 and 3 , but the temperature at the nozzle exit is higher in type 3 than type 2 . Therefore, the exit Mach number of type 2 is greater than type 3 .

\subsection{Microthrusters performance results}

Thrust, specific impulse, exit velocity and the mass flow rate are compared for three types of microthrusters. The total thrust is the sum of thrusts of each cell at the exit. The thrust is calculated using $F=\dot{m} V_{\text {exit }}$. The specific impulse is calculated as $I_{s p}=F /(\dot{m} g)$.

Quick comparison of the performance of three preceding microthruster systems is provided in Table 2. It is observed that the thrust and the specific impulse are highest in type 2. Therefore, a higher temperature would result in a higher thrust and specific impulse. Meanwhile, the minimum of thrust occurs in type 3 , where the heater plates are inserted in. In type 3 , the viscous effects of heater plates dominate the gas expansion and reduce the thrust due to significant viscous effects of the plates. In type 2 , the flow is heated and accelerated but there are no 
heater plates in the domain to increase the frictional forces. Hence, the performance parameters of type 2 are higher than the two others.

Table 2. Comparison of performance of three types of microthrusters

\begin{tabular}{|c|c|c|c|c|}
\hline & $\dot{m}[\mathrm{~g} / \mathrm{s}]$ & $V_{\text {exit }}[\mathrm{m} / \mathrm{s}]$ & $F[\mathrm{mN}]$ & $I_{s p}[\mathrm{~s}]$ \\
\hline \hline Type 1 & 12.3 & 980 & 11.1 & 106.7 \\
\hline Type 2 & 11.8 & 1310 & 14.7 & 144.7 \\
\hline Type 3 & 7.4 & 1290 & 7.1 & 121.3 \\
\hline
\end{tabular}

\subsection{Effects of temperature gradient}

Kundu et al. (2013) showed that there was a temperature gradient in a hydrogen proxide monopropellant microthruster at the chamber section. Therefore, in this Section, the effect of temperature gradient on the thruster performance is studied. Three cases of temperature gradients are implemented on the wall of micropropulsion device type 2 and on the heater plates of microthruster type 3 (see Table 3 ).

Table 3. Comparison of performance of three types of microthrusters

\begin{tabular}{|c|c|c|c|}
\hline $\begin{array}{c}\text { Type of } \\
\text { microthruster }\end{array}$ & Gradient case & $\begin{array}{c}\text { Temperature at the beginning } \\
\text { of wall/heater plates }[\mathrm{K}]\end{array}$ & $\begin{array}{c}\text { Temperature at end of } \\
\text { wall/heater plates [K] }\end{array}$ \\
\hline \hline \multirow{3}{*}{ Type 2/type 3 3} & 300 & 500 \\
\cline { 2 - 4 } & Linear: 1 & 300 & 700 \\
\cline { 2 - 4 } & Linear: 2 & 300 & 900 \\
\hline
\end{tabular}

Grid/PPC studies have been carefully carried out for all gradient cases. In this Section, only the results obtained from simulation are presented. Temperature gradients of microthrusters 2 and 3 without the gradient and with the gradient (case 3) are depicted in Fig. 7.

(a)

$T[\mathrm{~K}]$ :

$\begin{array}{cccccccccccccc}2 & 3 & 4 & 5 & 6 & 7 & 8 & 9 & 10 & 11 & 12 & 13 & 14 \\ 162 & 223 & 285 & 346 & 408 & 469 & 531 & 592 & 654 & 715 & 777 & 838 & 900\end{array}$

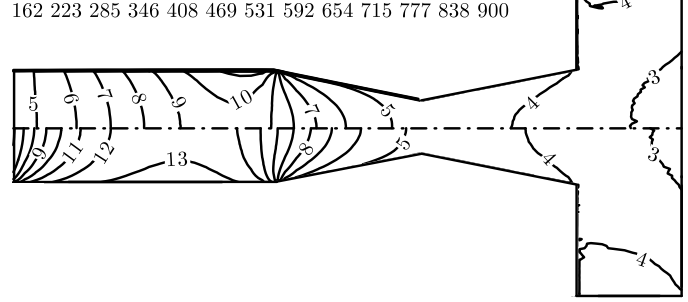

(b)

$T[\mathrm{~K}]$ :

$\begin{array}{llllllllllllll}2 & 3 & 4 & 5 & 6 & 7 & 8 & 9 & 10 & 11 & 12 & 13 & 14 & 15\end{array}$ $157214271329386443500557614671729786843 \quad 900$

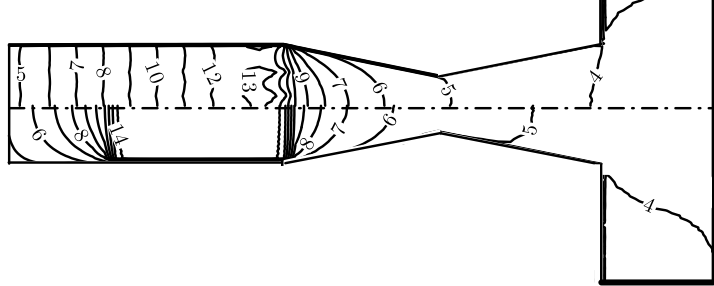

Fig. 7. Comparison of temperature contours for gradient case 3 and constant temperature: (a) gradient case (up), constant pre-heated wall (900 K) (bottom) for microthruster 2, (b) gradient case (up), constant pre-heated plate $(900 \mathrm{~K})$ (bottom) for microthruster 2

It is observed that the exit temperature of microthruster 2 is slightly larger than the exit temperature of microthruster 2 with gradient case 3 . The same temperature behavior is seen for thruster type 3 . Table 4 provides a quick review of the microthrusters performance.

It is demonstrated that temperature gradient case 3 has a higher thrust and specific impulse for both types of microthrusters (type 2 and 3 ). 
Table 4. Performance comparison of three types of microthrusters

\begin{tabular}{|c|c|c|c|c|c|}
\hline $\begin{array}{c}\text { Type of } \\
\text { microthruster }\end{array}$ & Gradient case & $\begin{array}{c}\text { Mass flow } \\
\text { rate }\left[\mathrm{gs}^{-1}\right]\end{array}$ & $\begin{array}{c}\text { Exit velocity } \\
{\left[\mathrm{ms}^{-1}\right]}\end{array}$ & $\begin{array}{c}\text { Thrust } \\
{[\mathrm{mN}]}\end{array}$ & $\begin{array}{c}\text { Specific } \\
\text { impulse }[\mathrm{s}]\end{array}$ \\
\hline \hline \multirow{3}{*}{2} & Linear: 1 & 12.1 & 1030 & 12.3 & 119.7 \\
\cline { 2 - 6 } & Linear: 2 & 12 & 1211 & 13.7 & 130.4 \\
\cline { 2 - 6 } & Linear: 3 & 11.7 & 1305 & 14.6 & 142.3 \\
\hline \multirow{3}{*}{3} & Linear: 1 & 6.85 & 1003 & 4.41 & 85.1 \\
\cline { 2 - 6 } & Linear: 2 & 6.9 & 1111 & 5.4 & 99.8 \\
\cline { 2 - 6 } & Linear: 3 & 7.2 & 1263 & 6.8 & 118.5 \\
\hline
\end{tabular}

\section{Performance comparison with/without temperature gradient}

Figure 8 compares the thrust, specific impulse, mass flow rate and the exit velocity of all types of microthrsueters with the constant temperature and with temperature gradient cases.
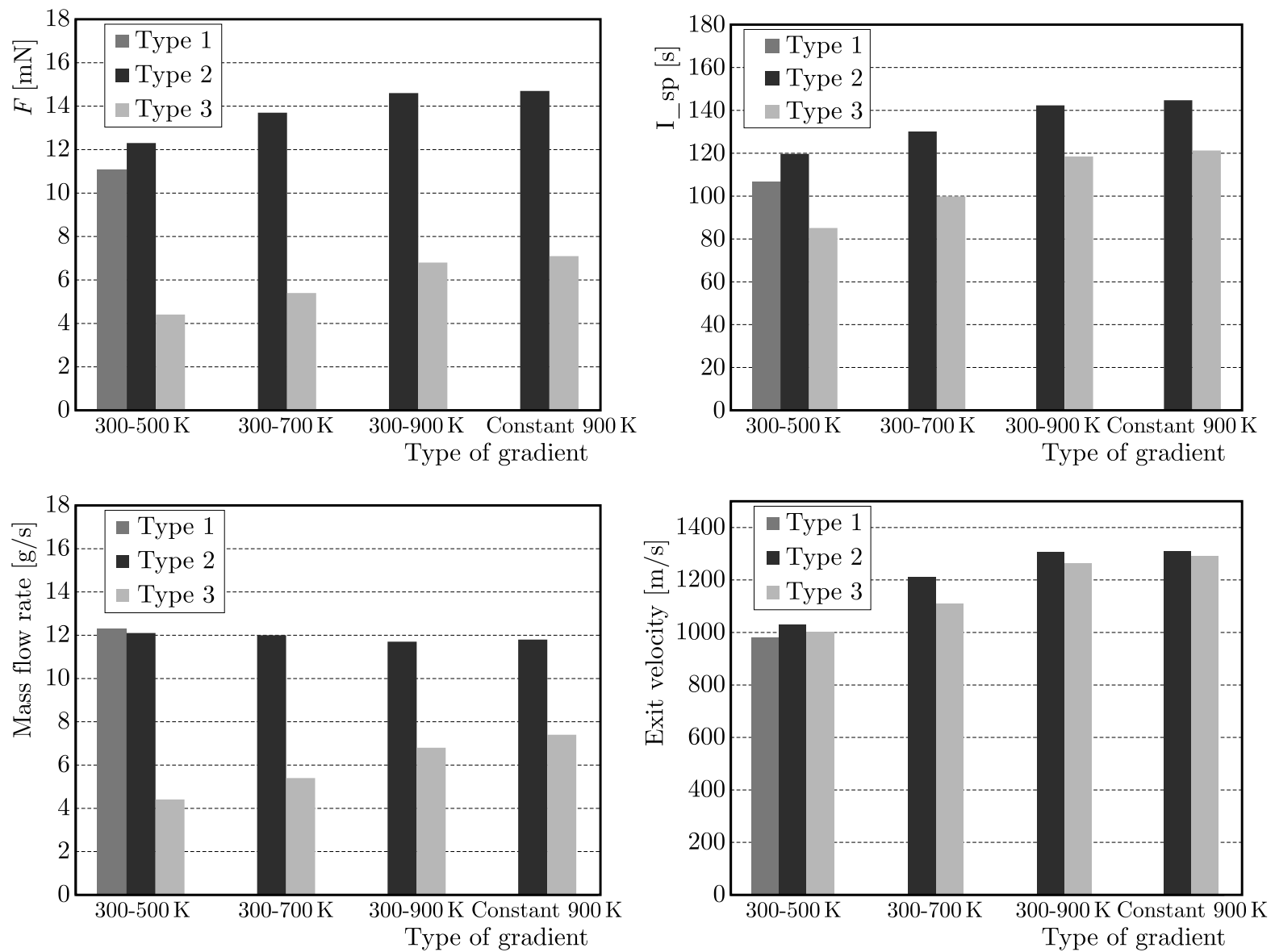

Fig. 8. Comparison of performance parameters of three types of microthrusters with constant pre-heated walls/plates and with gradient cases

It is observed that the highest thrust and specific impulse occurs in microthruster 2 with the constant wall temperature of $900 \mathrm{~K}$. Insertion of pre-heated plates into microthruster 3 decreases the thrust. Pre-heated plates/walls increase the exit velocity. However, by implementing a higher temperature gradient to walls/plates, the microthrusters performance is elevated. 


\section{Conclusion}

A DSMC/NS solver has been used in this paper. Accuracy of simulations has been first verified by simulation of a micronozzle. Then the flow inside three different microthruster system configurations has been simulated. A cold gas microthruster as type 1, pre-heated wall propulsion device as type 2, and a thruster with pre-heat plates inside the domain as type 3 have been simulated. It is observed that an increase in the fluid temperature accelerates the flow. Insertion of pre-heated plates inside the domain increases pressure loss, whereas heating up the walls of the thruster does not increase the frictional forces and, consequently, does not increase the pressure loss. It is demonstrated that type 2 has higher performance parameters than type 1 and 3. Also, temperature gradients have been implemented into the pre-heated walls and plates. It is observed that a higher gradient would elevate the thrust and specific impulse. Among all simulated cases, microthruster of type 2 with constant pre-heated walls (no temperature gradient) has better performance than all other cases. Microthruster 2 benefits from heating up which accelerates the flow, whereas it does not suffer from the frictional forces caused by the heater plates in type 3 .

\section{References}

1. Alexeenko A., Levin D., Gimelshein S., Collins R., Markelov G., 2002, Numerical simulation of high-temperature gas flows in a millimeter-scale thruster, Journal of Thermophysics and Heat Transfer, 16, 1, 10-16

2. BAYT R.L., 1999, Analysis, fabrication and testing of a MEMS-based micropropulsion system, $\mathrm{PhD}$, Aerospace Computational Design Laboratory, Department of Aeronautics and Astronautics, Massachusetts Institute of Technology

3. BiRd G., 2007, Sophisticated DSMC, Notes Prepared for a Short Course at the DSMC07 Meeting, Santa Fe, USA

4. BIRD G.A., 1994, Molecular Gas Dynamics and the Direct Simulation of Gas Flows, USA, Oxford Science Publications

5. Boyd I.D., Chen G., Candler G.V., 1995, Predicting failure of the continuum fluid equations in transitional hypersonic flows, Physics of Fluids, 7, 1, 210-219

6. Boyd I.D., Penko P.F., Meissner D.L., DeWitt K.J., 1992, Experimental and numerical investigations of low-density nozzle and plume flows of nitrogen, AIAA Journal, 30, 10, 2453-2461

7. Darbandi M., Roohi E., 2011, Study of subsonic-supersonic gas flow through micro/nanoscale nozzles using unstructured DSMC solver, Microfluidics and Nanofluidics, 10, 2, 321-335

8. GAD-EL-HAK M., 2005a, MEMS: Applications, USA, CRC Press

9. GAD-EL-HAK M., 2005b, MEMS: Introduction and Fundamentals, USA, CRC Press

10. Hadjiconstantinou N.G., 2000, Analysis of discretization in the direct simulation Monte Carlo, Physics of Fluids, 12, 10, 2634-2638

11. Hitt D.L., Zakrzwski C.M., Thomas M.A., 2001, MEMS-based satellite micropropulsion via catalyzed hydrogen peroxide decomposition, Smart Materials and Structures, 10, 6, 1163

12. Ivanov M., Markelov G., Ketsdever A., Wadsworth D., 1999, Numerical study of cold gas micronozzle flows, 37th Aerospace Sciences Meeting and Exhibit, Reno, NV, U.S.A., American Institute of Aeronautics and Astronautics

13. Jafryt Y., Beukelt J.V., 1994, Investigation of nozzle and plume expansions of a small helium thruster, Rarefied Gas Dynamics: Space Science and Engineering, 160, 136 
14. Janson S.W., Helvajian H., Hansen W.W., Lodmell J., 1999, Microthrusters for nanosatellites, Second International Conference on Integrated Micro Nanotechnology for Space Applications, Pasadena

15. Karniadakis G.E., Beskok A., Aluru N., 2006, Microflows and Nanoflows: Fundamentals and Simulation, Springer New York

16. Kundu P., Sinha A.K., Bhattacharyya T.K., Das S., 2013, Nanowire embedded hydrogen peroxide monopropellant MEMS thruster, Journal of Microelectromechanical Systems, 22, 2, 406-417

17. Le M., Hassan I., Esmail N., 2006, DSMC simulation of subsonic flows in parallel and series microchannels, Journal of Fluids Engineering, 128, 6, 1153-1163

18. LE N.T., Rooni E., 2015, A new form of the second-order temperature jump boundary condition for the low-speed nanoscale and hypersonic rarefied gas flow simulations, International Journal of Thermal Sciences, 98, 51-59

19. Le N.T., White C., Reese J.M., Myong R.S., 2012, Langmuir-Maxwell and Langmuir-Smoluchowski boundary conditions for thermal gas flow simulations in hypersonic aerodynamics, International Journal of Heat and Mass Transfer, 55, 19, 5032-5043

20. Liou W., FAng Y., 2000, Implicit boundary conditions for direct simulation Monte Carlo method in MEMS flow predictions, CMES - Computer Modeling in Engineering and Sciences, 1, 4, 119-128

21. Liu M., Zhang X., Zhang G., Chen Y., 2006, Study on micronozzle flow and propulsion performance using DSMC and continuum methods, Acta Mechanica Sinica, 22, 5, 409-416

22. Mihailovic M., Mathew T., Creemer J., Zandbergen B., Sarro P., 2011, MemS silicon-based resistojet micro-thruster for attitude control of nano-satellites, Solid-State Sensors, Actuators and Microsystems Conference (TRANSDUCERS), Beijing, IEEE: 262-265

23. Milligan M.W., 1964, Nozzle characteristics in the transition regime between continuum and free molecular flow, AIAA Journal, 2, 6, 1088-1092

24. Moss J.N., Bird G.A., 2005, Direct simulation Monte Carlo simulations of hypersonic flows with shock interactions, AIAA Journal, 43, 12, 2565-2573

25. Nance R.P., Hash D.B., Hassan H., 1998, Role of boundary conditions in Monte Carlo simulation of microelectromechanical systems, Journal of Thermophysics and Heat Transfer, 12, 3, 447-449

26. O’Hare L., Lockerby D.A., Reese J.M., Emerson D.R., 2007, Near-wall effects in rarefied gas micro-flows: some modern hydrodynamic approaches, International Journal of Heat and Fluid Flow, 28, 1, 37-43

27. OPENFOAM, 2014), OPENFOAM: The Open Source CFD Toolbox

28. Osiander R., Darrin M.A.G., Champion J.L., 2005, MEMS and Microstructures in Aerospace Applications, USA, CRC Press

29. Pfeiffer M., Mirza A., Fasoulas S., 2013, A grid-independent particle pairing strategy for DSMC, Journal of Computational Physics, 246, 28-36

30. Platt D., 2002, A monopropellant milli-Newton thruster system for attitude control of nanosatellites, AIAA/USU Conference on Small Stellites, Utah

31. RAE W.J., 1971, Some numerical results on viscous low-density nozzle flows in the slender-channel approximation, AIAA Journal, 9, 5, 811-820

32. Roohi E., Darbandi M., Mirjalili V., 2009, Direct simulation Monte Carlo solution of subsonic flow through micro/nanoscale channels, Journal of Heat Transfer, 131, 9, 092402

33. Rothe D.E., 1971, Electron-beam studies of viscous flow in supersonic nozzles, AIAA Journal, 9, $5,804-811$ 
34. Schwartzentruber T.E., Scalabrin L.C., Boyd I.D., 2007, A modular particlecontinuum numerical method for hypersonic non-equilibrium gas flows, Journal of Computational Physics, 225, 1, 1159-1174

35. Sun Z.-X., Li Z.-Y., He Y.-L., Tao W.-Q., 2009, Coupled solid (FVM)-fluid (DSMC) simulation of micro-nozzle with unstructured-grid, Microfluidics and Nanofluidics, 7, 5, 621-631

36. Sun Z.-X., TAng Z., He Y.-L., TaO W.-Q., 2011, Proper cell dimension and number of particles per cell for DSMC, Computers and Fluids, 50, 1, 1-9

37. Torre F.L., Kenjeres S., Kleijn C.R., Moerel J.-L.P., 2010, Effects of wavy surface roughness on the performance of micronozzles, Journal of Propulsion and Power, 26, 4, 655-662

38. WAng M., Li Z., 2004, Simulations for gas flows in microgeometries using the direct simulation Monte Carlo method, International Journal of Heat and Fluid Flow, 25, 6, 975-985

39. Wu J., Lee W., Lee F., Wong S., 2001, Pressure boundary treatment in internal gas flows at subsonic speed using the DSMC method, Rarefied Gas Dynamics: 22nd International Symposium, USA, American Institute of Physics, 408-416

40. Xie C., 2007, Characteristics of micronozzle gas flows, Physics of Fluids, 19, 3, 037102

Manuscript received December 31, 2016; accepted for print November 7, 2017 